Wie man leicht zeigen kann, bleibt die Form der zu (10) analogen Gleichung

$$
b \varphi=-\frac{1}{2} \sum_{l=1}^{6}\left(\frac{\partial^{2}}{\partial q l^{2}}+\frac{\partial^{2} \varrho^{(6)}}{\partial q l^{2}}\right) \varphi=\varepsilon \varphi
$$

erhalten, wenn man statt der Koordinaten $x_{1}, y_{1}, z_{1}$, $x_{2}, y_{2}, z_{2}$ die Koordinaten

$$
\begin{array}{ll}
x_{+}=\frac{1}{\sqrt{2}}\left(x_{1}+x_{2}\right), & x_{-}=\frac{1}{\sqrt{2}}\left(x_{1}-x_{2}\right), \\
y_{+}=\frac{1}{\sqrt{2}}\left(y_{1}+y_{2}\right), & y_{-}=\frac{1}{\sqrt{2}}\left(y_{1}-y_{2}\right), \\
z_{+}=\frac{1}{\sqrt{2}}\left(z_{1}+z_{2}\right), & z_{-}=\frac{1}{\sqrt{2}}\left(z_{1}-z_{2}\right)
\end{array}
$$

verwendet. [Der Übergang von

$$
\mathrm{r}_{1}=\left(x_{1}, y_{1}, z_{1}\right) \quad \text { und } \quad \mathrm{r}_{2}=\left(x_{2}, y_{2}, z_{2}\right)
$$

$\mathrm{zu}$

$$
\mathfrak{r}_{+}=\left(x_{+}, y_{+}, z_{+}\right) \quad \text { und } \quad \mathfrak{r}_{-}=\left(x_{-}, y_{-}, z_{-}\right)
$$

stellt eine Drehung im 6-dimensionalen Konfigurationsraum $R_{6}$ dar, gegen die (24) invariant ist.] Die Zweielektronen-Ortsfunktionen $\varphi$ müssen hinsichtlich der Vertauschung der Elektronen 1 und 2 symmetrisch (Singulettfunktionen) oder antisymmetrisch (Triplettfunktionen) sein. Der Vorteil der Koordinaten $\mathfrak{r}_{+}, \mathfrak{r}_{-}$ gegenüber $r_{1}, r_{2}$ besteht nun darin, daß $\varphi$ im ersten Falle eine gerade, im zweiten eine ungerade Funktion von allen Komponenten von $\mathfrak{r}_{-}$ist. Die Funktion $\varphi$ braucht also nur in $1 / 8$ des gesamten $R_{6}$ betrachtet zu werden; sind noch ein bzw. zwei bzw. drei Spiegelebenen vorhanden, welche die $x$-, $y$ - oder $z$-Achse des geometrischen Raums bei geeigneter Wahl des Koordinatensystems und damit auch entsprechend die $x_{+}^{-}, y_{+}-$oder $z_{+}$-Achse rechtwinklig im Koordinaten-

\section{Untersuchung frischer Fallout-Proben mit dem Ge(Li)-Halbleiterzähler}

\author{
H. KLös, R. MAY und H. SchNeIDER
}

I. Physikalisches Institut und Strahlenzentrum der Justus Liebig-Universität Gießen

(Z. Naturforschg. 21 a, 1502-1504 [1966] ; eingegangen am 18. Juli 1966)

Mit dem Ge(Li)-Halbleiterzähler wurde das $\gamma$-Spektrum frischer Fallout-Proben vom dritten chinesischen Kernwaffentest aufgenommen. Das Verhältnis der ${ }^{95} \mathrm{Zr}$-Linien bei 723 $\mathrm{keV}$ und $757 \mathrm{keV}$ wurde bestimmt. Aus dem Zirkon-NiobAktivitätsverhältnis kann das Alter der Probe errechnet werden.

Bei früheren Messungen wurde das $\gamma$-Spektrum langlebiger Radionuklide untersucht, die bei Kernwaffenversuchen entstanden sind ${ }^{1}$. Die dritte Explosion einer chinesischen Atombombe am 9. 5. 1966 ermöglichte es, auch kurzlebige $\gamma$-Strahler im Fallout nachzuweisen.

1 H. Klös, R. MAY u. H. Schneider, Z. Naturforschg. 21 a, 363 [1966]. ursprung schneiden, so reduziert sich die Betrachtung auf $1 / 16$ bzw. $1 / 32$ bzw. $1 / 64$ des $R_{6}$.

Die bei weitem einfachste Behandlung der SchröDinGER-Gleichung ist dann möglich, wenn sich die partielle Differentialgleichung, in entsprechenden Separationskoordinaten ausgedrückt, in mehrere gewöhnliche Differentialgleichungen separieren läßt. Leider ist eine solche Separation aber bisher nur bei Einelektronensystemen und auch dort nur bei Atomen (wasserstoffähnliche Atome) und zweiatomigen Molekülen $\left(\mathrm{H}_{2}^{+}\right.$, $\mathrm{HHe}^{++}$usw.) gelungen. $\mathrm{Da} ß$ die Größe $\varrho$ ein Schritt hin zu den vielleicht existierenden, aber noch unbekannten Separationskoordinaten bei anderen Systemen sein könnte, läßt die folgende Überlegung vermuten. Bei wasserstoffähnlichen Atomen (Atome mit der Kernladungszahl $Z$ und $N=1$ ) sind die Separationskoordinaten bekanntlich die sphärischen Koordinaten $r, \vartheta, \varphi^{\prime}$; wie man sieht, ist aber dort $\varrho=Z r$.

Im Falle der zweiatomigen Moleküle AB mit $N=1$ gelingt die Separation in elliptischen Koordinaten

$$
\xi=\frac{r_{\mathrm{A}}+r_{\mathrm{B}}}{R}, \quad \eta=\frac{r_{\mathrm{A}}-r_{\mathrm{B}}}{R}, \quad \varphi^{\prime}
$$

$(R=$ Kernabstand $\mathrm{A}-\mathrm{B})$. Es ist aber dort

$$
\varrho=Z_{\mathrm{A}} r_{\mathrm{A}}+Z_{\mathrm{B}} r_{\mathrm{B}}=\frac{1}{2} R\left(Z_{\mathrm{A}}+Z_{\mathrm{B}}\right) \xi+\frac{1}{2} R\left(Z_{\mathrm{A}}-Z_{\mathrm{B}}\right) \eta,
$$

d.h. $\varrho$ ist eine Linearkombinaiton von Separationskoordinaten! Im Falle der Atome und symmetrischen zweiatomigen Moleküle $\left(Z_{\mathrm{A}}=Z_{\mathrm{B}}\right)$ ist es sogar bis auf einen konstanten Faktor eine Separationskoordinate selbst. Im Falle der Atome ist dieser konstante Faktor die Kernladungszahl $Z$, im Falle der symmetrischen zweiatomigen Moleküle hat er den Wert

$$
\frac{1}{2} R\left(Z_{\mathrm{A}}+Z_{\mathrm{B}}\right)=R Z,
$$

da hier $Z_{\mathrm{A}}=Z_{\mathrm{B}}=Z$ ist.
Zwar stieg bei uns die abgelagerte Gesamt- $\beta$-Aktivität während weniger Tage nur auf den Maximalwert $0,8 \mathrm{nCi} / \mathrm{m}^{2}$ Tag am 23. 5. 1966 an (Jahresmittelwert 1963: $1 \mathrm{nCi} / \mathrm{m}^{2} \mathrm{Tag}$ ), jedoch gelang es trotzdem, bei Meßzeiten bis zu 50 Stunden $\gamma$-Spektren mit ausreichender Genauigkeit aufzunehmen. Insgesamt wurden 8 Meßreihen durchgeführt, wobei die Ergebnisse stets reproduzierbar waren, abgesehen von Änderungen des Spektrums, die auf den radioaktiven Zerfall kurzlebiger Nuklide zurückzuführen sind. Verwendet wurde ein $\mathrm{Ge}(\mathrm{Li})$-Halbleiterzähler 100-LDG-4000-310/Kryostat (Solid State Radiation, Inc.) mit $4 \mathrm{~mm}$ dicker Feldzone und $1 \mathrm{~cm}^{2}$ Zählfläche. Angeschlossen war das Verstärkersystem $101 \mathrm{XL} / 201$ (ORTEC). Die verstärkten Impulse wurden einem 1024-Kanal-Impulshöhen-Analysator ND-150 (Nuclear Data) zugeführt. Der Detektor war mit Bleiziegeln und Betonsteinen gegen die Umgebungsstrahlung abgeschirmt.

\section{Meßergebnisse}

Wie die Abb. 1 bis 3 zeigen, konnten neben den bereits mit dem $\mathrm{Ge}(\mathrm{Li})$-Detektor ${ }^{1}$ gefundenen Radio- 
nukliden ${ }^{144} \mathrm{Ce},{ }^{125} \mathrm{Sb},{ }^{106} \mathrm{Rh},{ }^{137} \mathrm{Cs}$ und ${ }^{57} \mathrm{Co}$ zahlreiche kurzlebige Spaltprodukte identifiziert werden.

${ }^{147} \mathrm{Nd}$ zeigt eine starke $\gamma$-Linie bei $91 \mathrm{keV}$ und eine schwache bei $531 \mathrm{keV}$; in Abb. 2 wird ein weiterer Photopeak bei $198 \mathrm{keV}$ erkennbar, der anfangs von dem benachbarten starken Peak bei $208 \mathrm{keV}$ verdeckt wird. Es ist zu erwarten, daß zu dem Maximum bei $198 \mathrm{keV}$ auch das Spaltprodukt ${ }^{156} \mathrm{Eu}$ einen Beitrag liefert. In Abb. 1 ist ferner bei $40 \mathrm{keV}$ die PromethiumRöNTGEN-Strahlung festzustellen.

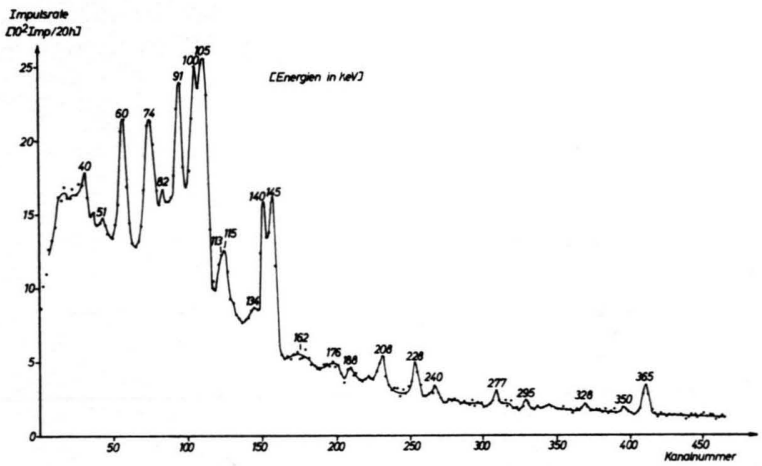

Abb. 1. $\gamma$-Spektrum einer Fallout-Probe, gemessen am 26. 5 . 1966 mit dem $\mathrm{Ge}(\mathrm{Li})$-Halbleiterzähler, je zwei Kanäle zusammengefaßt. (Untergrund nicht abgezogen.)

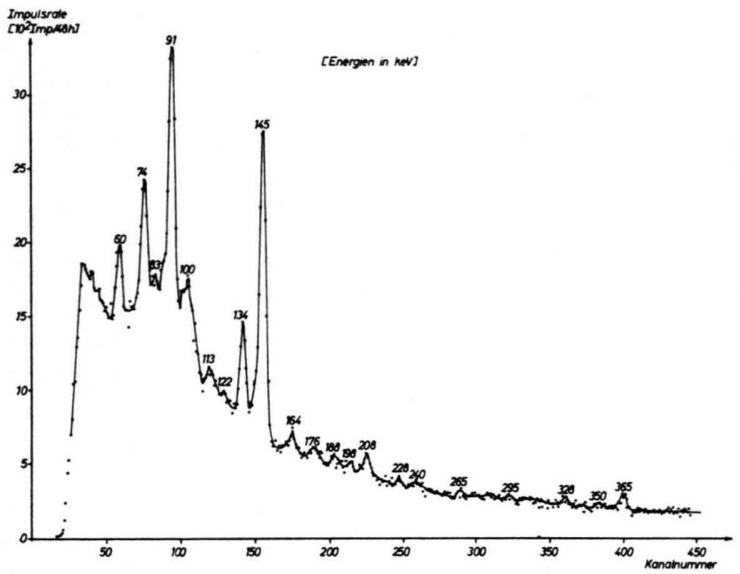

Abb. 2. $\gamma$-Spektrum derselben Probe, gemessen am 3. und 4.6. 1966.

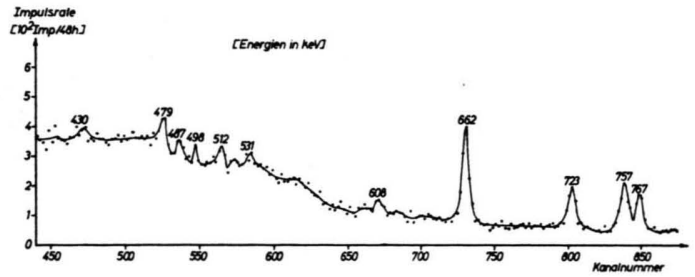

Abb. 3. Fortsetzung von Abb. 2; oberer Energiebereich, je zwei Kanäle zusammengefaßt. (Untergrund nicht abgezogen.)

\begin{tabular}{|c|c|c|c|}
\hline $\begin{array}{c}\text { Abb. I } \\
\text { Energie } \\
(\mathrm{keV})\end{array}$ & \begin{tabular}{|c|} 
Abb. 2, 3 \\
Energie \\
$(\mathrm{keV})$
\end{tabular} & Nuklid & Halbwertszeit \\
\hline 40 & - & Pm-Rö & $11 \mathrm{~d}\left({ }^{147} \mathrm{Nd}\right)$ \\
\hline \multirow[t]{2}{*}{51} & - & ${ }^{214} \mathrm{~Pb}$ & - \\
\hline & & ${ }^{239} \mathrm{~Np}$ & $2,3 \mathrm{~d}$ \\
\hline 60 & 60 & ${ }^{237} \mathrm{U}$ & $6,7 \mathrm{~d}$ \\
\hline 74 & 74 & $\mathrm{~Pb}-\mathrm{Rö}(\mathrm{L})$ & - \\
\hline \multirow[t]{2}{*}{82} & 83 & $\mathrm{~Pb}-\mathrm{Rö}(\mathrm{M})$ & - \\
\hline & & ${ }^{144} \mathrm{Ce}$ & $285 \mathrm{~d}$ \\
\hline 91 & 91 & ${ }^{147} \mathrm{Nd}$ & $11 \mathrm{~d}$ \\
\hline 100 & 100 & Np-Rö(L) & $6,7 \mathrm{~d}\left({ }^{237} \mathrm{U}\right)$ \\
\hline 105 & - & ${ }^{239} \mathrm{~Np}+\mathrm{Pu}-\mathrm{Rö}(\mathrm{L})$ & $2,3 \mathrm{~d}$ \\
\hline 113 & 113 & Np-Rö(M) & $6,7 \mathrm{~d}\left({ }^{237} \mathrm{U}\right)$ \\
\hline 115 & - & Pu-Rö(M) & $2,3 \mathrm{~d}\left({ }^{239} \mathrm{~Np}\right)$ \\
\hline- & 122 & ${ }^{57} \mathrm{Co}$ & $270 \mathrm{~d}$ \\
\hline 134 & 134 & ${ }^{144} \mathrm{Ce}$ & $285 \mathrm{~d}$ \\
\hline 140 & - & ${ }^{99} \mathrm{Mo}$ & $66 \mathrm{~h}$ \\
\hline 145 & 145 & ${ }^{141} \mathrm{Ce}$ & $33 \mathrm{~d}$ \\
\hline 162 & 164 & ${ }^{237} \mathrm{U}$ & $6,7 \mathrm{~d}$ \\
\hline 176 & 176 & ${ }^{125} \mathrm{Sb}$ & $2,0 \mathrm{a}$ \\
\hline 188 & 188 & ${ }^{226} \mathrm{Ra}$ & - \\
\hline- & 198 & ${ }^{147} \mathrm{Nd}$ & $11 \mathrm{~d}$ \\
\hline 208 & 208 & ${ }^{237} \mathrm{U}$ & $6,7 \mathrm{~d}$ \\
\hline 228 & 228 & ${ }^{239} \mathrm{~Np}$ & $2,3 \mathrm{~d}$ \\
\hline 240 & 240 & $(\mathrm{RaB})$ & - \\
\hline - & 265 & ${ }^{237} \mathrm{U}$ & $6,7 \mathrm{~d}$ \\
\hline 277 & - & ${ }^{239} \mathrm{~Np}$ & $2,3 \mathrm{~d}$ \\
\hline 295 & 295 & $(\mathrm{RaB})$ & - \\
\hline 328 & 328 & ${ }^{140} \mathrm{La}$ & $40 \mathrm{~h}\left({ }^{140} \mathrm{Ba}: 13 \mathrm{~d}\right)$ \\
\hline 350 & 350 & $(\mathrm{RaB})$ & - \\
\hline \multirow[t]{13}{*}{365} & 365 & $131 \mathrm{~J}$ & $8,1 \mathrm{~d}$ \\
\hline & 430 & ${ }^{125} \mathrm{Sb}$ & $2,0 \mathrm{a}$ \\
\hline & 479 & ${ }^{7} \mathrm{Be}$ & $54 \mathrm{~d}$ \\
\hline & 487 & ${ }^{140} \mathrm{La}$ & $40 \mathrm{~h}\left({ }^{140} \mathrm{~B}: 13 \mathrm{~d}\right)$ \\
\hline & 498 & ${ }^{103} \mathrm{Ru}$ & $40 \mathrm{~d}$ \\
\hline & 512 & ${ }^{106} \mathrm{Rh}$ & $30 \mathrm{~s}\left({ }^{106} \mathrm{Ru}: 1,0 \mathrm{a}\right)$ \\
\hline & & Positr.-Vernicht. & - \\
\hline & 531 & ${ }^{147} \mathrm{Nd}$ & - \\
\hline & 608 & $(\mathrm{RaC})$ & - \\
\hline & 662 & ${ }^{137} \mathrm{Cs}$ & 26,6 a \\
\hline & 723 & ${ }^{95} \mathrm{Zr}$ & $65 \mathrm{~d}$ \\
\hline & 757 & ${ }^{95} \mathrm{Zr}$ & $65 \mathrm{~d}$ \\
\hline & 767 & ${ }^{95} \mathrm{Nb}$ & $35 \mathrm{~d}$ \\
\hline
\end{tabular}

Tab. 1. Gemessene $\gamma$-Energiewerte, zugehörige Strahler und deren Halbwertszeit. [Rö $(\mathrm{L})=$ RöNTGEN-Strahlung; Ubergänge aus der L-Schale; Rö $(M)=$ Röntgen-Strahlung; Ubergänge aus der M-Schale.] 
In Abb. 1 tritt ein Photopeak bei $140 \mathrm{keV}$ auf, der von ${ }^{99}$ Mo stammt, wegen dessen kurzer Halbwertszeit von $66 \mathrm{~h}$ aber bald verschwindet. Bei $145 \mathrm{keV}$ ist die $\gamma$-Linie des ${ }^{141} \mathrm{Ce}$ zu erkennen. ${ }^{131} \mathrm{~J}$ wird durch seinen stärksten Übergang bei $365 \mathrm{keV}$ nachgewiesen.

${ }^{140} \mathrm{La}$, das kurzlebige (Halbwertszeit $T=40 \mathrm{~h}$ ) Folgeprodukt von ${ }^{140} \mathrm{Ba} \quad(T=13 \mathrm{~d})$, verursacht schwache Photopeaks bei $328 \mathrm{keV}$ und $487 \mathrm{keV}$. Dagegen konnte ${ }^{140} \mathrm{Ba}$ selbst, dessen stärkster Übergang bei etwa 537 $\mathrm{keV}$ liegt, nicht direkt nachgewiesen werden.

Die $498 \mathrm{keV}-\gamma$-Linie stammt vom ${ }^{103} \mathrm{Ru} .{ }^{95} \mathrm{Zr}$ verursacht zwei deutliche Photopeaks bei $723 \mathrm{keV}$ und $757 \mathrm{keV}$, das Folgeprodukt ${ }^{95} \mathrm{Nb}$ einen Photopeak bei $767 \mathrm{keV}$. Aus drei Messungen wurde das Verhältnis der ${ }^{95} \mathrm{Zr}$-Linie bei $757 \mathrm{keV}$ zur ${ }^{95} \mathrm{Zr}$-Linie bei $723 \mathrm{keV}$ zu 1,35 $\pm 0,13$ bestimmt. Es sind genauere Messungen mit Standardpräparaten geplant, zumal die Werte in der Literatur stark voneinander abweichen und z. T. mit kleiner als eins angegeben sind.

Schon nach dem ersten chinesischen Kernwaffentest schlossen wir aus der zeitlichen Änderung der Gesamt$\beta$-Aktivität, daß neben den Spaltprodukten erhebliche Mengen neutronenaktivierter Substanzen vorliegen müssen. Hierfür wurden Halbwertszeiten von 1,3 bzw. 2,5 Tagen gemessen ${ }^{2}$. $\mathrm{Zu}$ der letzteren paßt das Radionuklid ${ }^{239} \mathrm{~Np}(T=2,346 \mathrm{~d})$; die geringe Abweichung ist durch die Beimischung von ${ }^{99} \mathrm{Mo}(T=2,75 \mathrm{~d}) \mathrm{zu}$ erklären. ${ }^{239} \mathrm{~Np}$ konnte nun auch im $\gamma$-Spektrum (Abb. 1) gefunden werden, und zwar durch Photopeaks bei 105 $\mathrm{keV}, 228 \mathrm{keV}$ und $277 \mathrm{keV}$; weiterhin ist ein Beitrag zum Peak bei $51 \mathrm{keV}$ zu erwarten. Plutonium-RöntgenStrahlung tritt ebenfalls bei $105 \mathrm{keV}$ und bei $115 \mathrm{keV}$ auf. In Abb. 2 sind diese Peaks bis auf einen geringen Rest bei $228 \mathrm{keV}$ nicht mehr ausgeprägt.

2 R. May u. H. Schneider, Z. Naturforschg. 20 a, 166 [1965].
Weiterhin wurde das neutronenaktivierte Nuklid ${ }^{237} \mathrm{U}$ mit $\gamma$-Linien bei $60 \mathrm{keV}, 164 \mathrm{keV}, 208 \mathrm{keV}$ und $265 \mathrm{keV}$ gefunden; bei $100 \mathrm{keV}$ und $113 \mathrm{keV}$ wird die Röntgen-Strahlung des Neptuniums gemessen.

Die übrigen Photopeaks sind auf natürliche Radioaktivität zurückzuführen. Die $479 \mathrm{keV}$-Linie stammt von ${ }^{7} \mathrm{Be}$, das durch kosmische Höhenstrahlung in der Atmosphäre erzeugt wird und in unterschiedlicher Konzentration stets nachweisbar ist. ${ }^{226} \mathrm{Ra}$ bzw. seine Folgeprodukte ${ }^{214} \mathrm{~Pb}$ und ${ }^{214} \mathrm{Bi}$ verursachen Photopeaks bei $188 \mathrm{keV}, 240 \mathrm{keV}, 295 \mathrm{keV}, 350 \mathrm{keV}$ und $608 \mathrm{keV}$; auch ein Anteil am Maximum bei $51 \mathrm{keV}$ ist möglich. Die Röntgen-Strahlung des Bleis schließlich tritt bei $74 \mathrm{keV}$ und $83 \mathrm{keV}$ auf.

\section{Altersbestimmung aus dem Zirkon-Niob-Aktivitäts- verhältnis}

Die einzige sichere Methode zur Altersbestimmung von Spaltproduktgemischen besteht darin, das ZirkonNiob-Aktivitätsverhältnis der Probe zur Datierung der Explosion heranzuziehen. Das Verfahren ist anwendbar, solange Zirkon und Niob sich noch nicht im Gleichgewicht befinden. Hierzu kamen bisher Abklingmessungen, $\beta$ - $\gamma$-Koinzidenzmessungen ${ }^{3}$ oder chemische Trennung in Betracht. Wie Abb. 3 zeigt, können mit dem Halbleiterdetektor jedoch die $\gamma$-Linien von ${ }^{95} \mathrm{Zr}$ und ${ }^{95} \mathrm{Nb}$ getrennt werden, so daß aus den gemessenen Intensitäten das Alter errechnet werden kann.

Wir danken Herrn Prof. Dr. W. HANLE für sein förderndes Interesse und dem Bundesministerium für wissenschaftliche Forschung für Bereitstellung von Forschungsmitteln.

${ }^{3}$ R. MAY, Atomkernenergie 11, H. 7/8 [1966].

\section{Inelastic Electron Scattering from Levels with 7 to $15 \mathrm{MeV}$ Excitation Energy in ${ }^{24} \mathrm{Mg}$ and ${ }^{26} \mathrm{Mg}$}

\author{
O. Titze and E. Spamer
}

Institut für Technische Kernphysik der Technischen Hochschule Darmstadt

(Z. Naturforschg. 21 a, 1504-1506 [1966] ; received 22 July 1966)

Energy spectra of electrons scattered inelastically from ${ }^{24} \mathrm{Mg}$ and ${ }^{26} \mathrm{Mg}$ were measured. The electric or magnetic character of 22 transitions was deduced from studies of the angular distributions. Spins, parities and ground state radiation widths were determined for levels at $9,97,10.35,10.70$, and $10.93 \mathrm{MeV}$ in ${ }^{24} \mathrm{Mg}$.

Using the facilities of the Darmstadt $60 \mathrm{MeV}$ linear accelerator $^{1}$ we have studied inelastic electron scat-

1 F. Gudden, G. Fricke, H.-G. Clerc u. P. Brix, Z. Phys. 181, 453 [1964].

2 M. Stroetzel, Laborbericht 19, Institut für Technische Kernphysik der Technischen Hochschule Darmstadt 1965, unpublished. tering from isotopically enriched ${ }^{24} \mathrm{Mg}$ and ${ }^{26} \mathrm{Mg}$. The counting rates from a new five-detector system ${ }^{2}$ were summed up to give single spectra with good statistical accuracy (better than $1 \%$ ).

Although the level density in these nuclei is high for excitation energies $E_{x}>7 \mathrm{MeV}$, one selects in electron scattering experiments only those levels that have appreciable ground state radiation widths $\Gamma_{\gamma}{ }^{0}$. At low incident electron energies $E_{0}$ the number will be further reduced by the fact that the most probable transitions are M1 and E2.

For ${ }^{24} \mathrm{Mg}$ we resolve in the excitation energy range $E_{x}=7$ to $15 \mathrm{MeV}$ about 20 transitions as shown in Fig. 1. The only comparison possible is with the $180^{\circ}$ electron scattering experiment of BARBER et al. ${ }^{3}$ on natural $\mathrm{Mg}$ which shows two broad structures at 11

3 W. C. Barber, J. Goldemberg, G. A. Peterson u. Y. Torizuka, Nucl. Phys. 41, 461 [1963]. 